\title{
ASCA Observations of Narrow-Line Seyfert 1 Galaxies
}

\author{
K. Hayashida \\ Department of Earth 6 Space Science, Osaka University, 1-1 \\ Machikaneyama-cho, Toyonaka, Osaka, Japan
}

\begin{abstract}
ASCA observations of four narrow-line Seyfert 1 galaxies are presented. Among the four sources, two show $\mathrm{X}$-ray spectra consisting of soft and hard components. Rapid X-ray variability is observed in all four sources. We estimate the central black-hole mass of these sources and find indications that the apparent luminosities exceed the Eddington limit under some assumptions.
\end{abstract}

\section{Introduction}

Narrow-line Seyfert 1 (NLS1) galaxies are a class of Seyferts having both type 1 and type 2 characteristics in their optical spectra; their [O III]/H $\beta$ flux ratios are less than 3 , while their $\mathrm{H} \beta$ line widths are as narrow as $500-1500 \mathrm{~km} \mathrm{~s}^{-1}$. Although about $10 \%$ of optically selected Seyfert 1s belong to the NLS1 class, NLS1s appear more frequently $(16-50 \%)$ in soft X-ray selected samples. After the discovery of unique X-ray properties of the NLS1 IRAS 13224-3809 (Boller et al. 1993), many NLS1s have been observed with ROSAT. Those observations indicate that (1) NLS1s have generally steeper soft X-ray slopes than normal Seyfert 1s, and (2) many NLS1s show rapid X-ray variability (Boller et al. 1996).

IRAS 13224-3809 was also observed with $A S C A$, revealing its unique Xray spectrum which consists of two components: a soft component approximated by a $k T \approx 0.14 \mathrm{keV}$ blackbody model, and a power-law tail seen above $2 \mathrm{keV}$ (Otani et al. 1996). A similar spectrum, but with a weaker soft component, was also reported for RE $1034+39$ by Pounds et al. (1995). In this paper, we present $A S C A$ observations and our analysis of four NLS1s, Mrk 766, IRAS 13224, H0707-495, and IZw 1. Note some results on Mrk 766 and IRAS 13224 have been presented elsewhere (Otani et al. 1996; Leighly et al. 1996).

\section{X-ray Spectra}

Among the four sources, the spectra of Mrk 766 and I Zw 1 are basically fitted with a simple power-law model, with photon index $\Gamma=2.07 \pm 0.01$ and $\Gamma=$ $2.32 \pm 0.03$, respectively. The iron $\mathrm{K} \alpha$ emission line is detected at about $6.4 \mathrm{keV}$ in these two sources. Furthermore, Mrk 766 shows a warm-absorber feature.

On the other hand, H0707 (Fig. 1) has two distinct spectral components, resembling IRAS 13224 reported by Otani et al. (1996). We applied several models to fit the soft component in IRAS 13224 and H0707. An additional steep power-law model gives an unacceptable fit. A thin thermal-plasma model, 


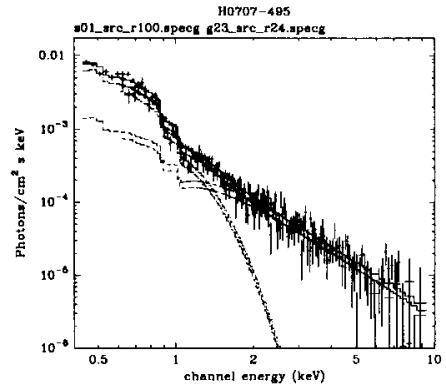

Figure 1. X-ray spectrum of H0707-495. Figure 2. $M_{B H}$ vs. $L_{x}$.

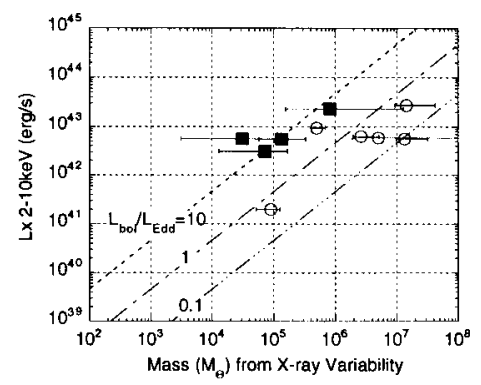

blackbody and disk blackbody gives a better fit, though a thin thermal model is not likely because of the low (less than $0.1 Z_{\odot}$ ) metal abundance derived. With disk blackbody model, we obtain the inner-disk temperature $k T_{i n}=107 \pm 3 \mathrm{eV}$ and $k T_{\text {in }}=189 \pm 14 \mathrm{eV}$ for IRAS 13224 and $\mathrm{H} 0707$, respectively. Assuming $H_{0}=75 \mathrm{~km} \mathrm{~s}^{-1} \mathrm{Mpc}^{-1}$ and disk inclination $\theta=0^{\circ}$, the inner radius of the disk $R_{\text {in }}=3.6 \times 10^{6} \mathrm{~km}$ and $R_{\text {in }}=3.4 \times 10^{5} \mathrm{~km}$, respectively. If they correspond to $3 R_{s}$ (where $R_{s}$ is the Schwarzshild radius), central black-hole masses of $M=$ $4.1 \times 10^{5} M_{\odot}$ and $M=3.8 \times 10^{4} M_{\odot}$, respectively, are obtained for these sources. Nevertheless, adopting these values, the total luminosity of the disk blackbody component $\left(3.1 \times 10^{44}\right.$ ergsec and $2.5 \times 10^{43} \mathrm{ergs} \mathrm{s}^{-1}$ for IRAS 13224 and $\mathrm{H} 0707$, respectively) exceeds the Eddington luminosity by about one order of magnitude.

Furthermore, we notice that $\mathrm{H} 0707$ also shows a spectral feature around $1 \mathrm{keV}$ that is similar to that reported for IRAS 13224 by Otani et al. (1996). Although the spectral feature in IRAS 13224 and H0707 is fitted with absorptionedge or notch model, we do not have a good candidate for the absorber body, unless we assume a blueshift of $0.2-0.3 c$ for the OVIII absorption edge. The photon index of the hard power-law component is $\Gamma=2.15 \pm 0.13$ and $\Gamma=$ $2.31 \pm 0.11$ for IRAS 13224 and H0707, respectively, although the value is affected by the model for the $1 \mathrm{keV}$ feature. Due to the low photon flux at higher energy, a statistically significant iron $\mathrm{K} \alpha$ line is not found in these two sources.

\section{X-ray Variability}

We find evidence for X-ray flux variability for all the four sources during their one or two-day observations. Mrk 766 and IZw 1 exhibit about factor-of-two variability with time scales down to thousands of seconds. IRAS 13224 and H0707, the sources with two-component spectra, show more enhanced variability of an order of magnitude with variability time scales as short as hundreds of seconds. In order to quantify the variability, we use a normalized power spectrum. density function. It is found that the variability power at $10^{-3} \mathrm{~Hz}$ is $15 \mathrm{~Hz}^{-1}$ (rms/int) ${ }^{2}$ both for $\mathrm{H} 0707$ and IRAS 13224. This is about factor of three larger than that of NGC 4051, which has been known as the Seyfert 1 with the most rapid variability. 
We have determined the relative time scale of variability for several Seyferts and also for Cyg X-1 (Hayashida et al. 1995). If we assume a linear scaling relation between the black-hole size and the variability time scale, we can estimate the black-hole mass by using Cyg X-1 as a reference point. The masses obtained with this method are $1.4 \times 10^{5} M_{\odot}, 3.1 \times 10^{4} M_{\odot}, 7.2 \times 10^{4} M_{\odot}$, and $8.2 \times 10^{5} M_{\odot}$ for Mrk 766, IRAS 13224, H0707, and I Zw 1, respectively. In Fig. 2, we plot black-hole mass estimated from the variability against $2-10 \mathrm{keV}$ X-ray luminosity, where Seyfert 1s (data from Ginga are denoted with open circles and the four NLS1s from $A S C A$ with solid squares). Using a bolometric correction of 27.2 , we find that the NLS1s have $L_{b o l} / L_{E d d} \approx 10$, while this ratio is $0.1-1$ for most of the Seyfert 1s, as indicated by Hayashida et al. (1995).

A correlation plot between the $0.4-1 \mathrm{keV}$ flux and $1-10 \mathrm{keV}$ flux indicates that the soft and the hard components in IRAS 13224 and H0707 vary roughly together. Preliminary correlation analysis indicates no statistically significant time lag between the two components.

\section{Central Black-Hole Mass and Emission Efficiency}

We estimated the central black-hole mass from two independent methods and found that apparent luminosity of these sources exceeds the Eddington limit by about one order of magnitude. Of course, the assumptions employed in both mass estimation methods might be wrong. The inner-disk radius might be smaller than $3 R_{s}$ for a Kerr metric, the simplified disk-radiation model might be inappropriate, or the scaling relation of the variability and the system size may break down.

Nevertheless, relativistic beaming is another solution. One of the explanation for the narrow line width in NLS1s is pole-on geometry, i.e., the broadline cloud motions are confined to the plane perpendicular to our line of sight. Since we know that radio-loud objects with pole on geometry exhibit relativistic beaming, we may speculate that the NLS1s are the radio-quiet equivalent of these sources. If the absorption structure around $1 \mathrm{keV}$ is real and corresponds to the blueshifted $\mathrm{O}$ VIII edge, this would provide further support of this idea. In this case, however, we may have to consider an origin of the soft component other than emission from the accretion disk.

We also note that the relatively smaller masses obtained for these four NLS1s can be responsible for the narrow line widths if the emission-line clouds are gravitationally bound to the central black hole.

\section{Analogy to Stellar Black-Hole Candidates}

As pointed out by Pounds et al. (1995), the two-component spectrum is analogous to the X-ray spectra of stellar black-hole candidates (SBHC) in their high state. The photon index of the power-law component is $\Gamma=2-3$ in high-state SBHCs, in contrast to $\Gamma=1.5-2$ in their low state (and also for typical Seyfert 1s). For RE $1034+39, \Gamma=2.6$ and for the four NLS1s analyzed by us $\Gamma$ lies between 2.0 and 2.3, in agreement with the values seen in high-state SBHCs.

However, we note that X-ray variability is enhanced particularly in the two-component spectrum NLS1s, in contrast to suppression of the variability in 
high-state SBHCs. Furthermore, disk blackbody fits to the soft component have resulted in the super-Eddington problem. Considering the completely different system sizes, it is reasonable to expect that the similarity to the high-state SBHCs is incomplete even if the NLS1s are high-state AGNs. Even in that case, comparison with SBHCs will be important to see the black-hole size effect.

\section{NLS1, Soft X-ray Excess, Steep Soft X-ray Spectrum AGNs}

The soft components of IRAS 13224 and H0707 might be regarded as extreme case of the soft X-ray excess found in a significant fraction of AGNs (e.g., Walter $\&$ Fink 1993). The relatively strong soft X-ray excess component of Mrk 841 and PG $1211+143$ observed with $A S C A$ has a $0.5 \mathrm{keV}$ intensity that is comparable to or smaller than that of the hard power-law component (Murakami et al. 1996). In contrast to this, the soft components of H0707 and IRAS 13224 has 5 to 10 times the intensity of the hard power-law component at $0.5 \mathrm{keV}$.

If the steep soft X-ray spectra of NLS1s observed with ROSAT are mainly due to an enhanced soft component, we had better interpret the narrow $\mathrm{H} \beta$ line width and soft $\mathrm{X}$-ray excess as having a close connection with each other. In fact, $\mathrm{PG} 1211+143$ has a relatively narrow $\mathrm{H} \beta$ line width (Murakami et al. 1996). If the soft $\mathrm{X}$-ray excess component originated in thermal emission from a thin disk, the pole-on geometry qualitatively gives this correlation. However, if the simple geometry is the only cause of the correlation, there should be more examples like IRAS 13224 and $\mathrm{H} 0707$.

There remain many questions on the nature of NLS1s, e.g., what is the origin of the soft and hard components, what determines the strength of the soft components, whether or not there is high-low transition of the X-ray spectrum of NLS1s as for SBHCs, what is the origin of $1 \mathrm{keV}$ absorption structure, and what is the iron $\mathrm{K} \alpha$ line like in the two-component spectrum sources? The $A S C A$ response is nicely designed for NLS1s, particularly for two-component type spectra. We hope further observations of NLS1s with $A S C A$ will answer some of these questions.

\section{References}

Boller, Th., et al. 1993, A\&A, 279, 53.

Boller, Th., Brandt, W. N., \& Fink, H. 1996, A\&A, 305, 53.

Hayashida, K., et al. 1995, ApJ, submitted.

Leighly, K.M., et al. 1996, ApJ, in press.

Murakami, S., et al. 1996, in preparation

Otani, C., Kii, T., \& Miya, K. 1996, in MPE Report 263, 491.

Pounds, K. A., Done, C., \& Osborne, J. P. 1995, MNRAS, 277, L5.

Walter, R., \& Fink, H. 1993, A\&A, 274, 105. 